\title{
Magdalen Laundries : enjeu des droits de l'homme et responsabilité publique
}

\section{Nathalie Sebbane}

\section{OpenEdition}

1 Journals

\section{Édition électronique}

URL : http://journals.openedition.org/etudesirlandaises/2964

DOI : $10.4000 /$ etudesirlandaises. 2964

ISSN : 2259-8863

\section{Éditeur}

Presses universitaires de Caen

\section{Édition imprimée}

Date de publication : 30 juin 2012

Pagination : 41-56

ISSN : 0183-973X

\section{Référence électronique}

Nathalie Sebbane, «Magdalen Laundries : enjeu des droits de l'homme et responsabilité publique »,

Études irlandaises [En ligne], 37-1 | 2012, mis en ligne le 30 juin 2014, consulté le 10 décembre 2020. URL : http://journals.openedition.org/etudesirlandaises/2964; DOI : https://doi.org/10.4000/ etudesirlandaises.2964

\section{(c) (†) (9)}

Études irlandaises est mise à disposition selon les termes de la Licence Creative Commons Attribution - Pas d'Utilisation Commerciale - Partage dans les Mêmes Conditions 4.0 International. 


\title{
Magdalen Laundries : enjeu des droits de l'homme et responsabilité publique
}

\author{
Nathalie Sebbane \\ Université Sorbonne Nouvelle - Paris 3
}

\section{Résumé}

Le cas des Magdalen Laundries, institutions gérées par des congrégations religieuses dans lesquelles furent enfermées des centaines de femmes depuis le dix-neuvième siècle, fait l'objet d'une attention particulière depuis les années 1990. Or, depuis que le scandale a éclaté, seule l'Église catholique irlandaise a été mise en cause. On lui reproche d'avoir fait subir des traitements inhumains à ces femmes et d'avoir bénéficié financièrement du travail de blanchisserie qu'elles effectuaient dans ces institutions. En 2010, une organisation, Justice For Magdalens, a demandé à la Commission des Droits de l'Homme irlandaise de diligenter une enquête afin que soit déterminé le degré de responsabilité de l'État et que les anciennes pensionnaires et leurs enfants obtiennent réparation. La Commission a examiné la requête de Justice For Magdalens mais a décidé de ne pas diligenter d'enquête, faute de pouvoir véritablement se prononcer sur la question des droits de l'homme ${ }^{1}$. En revanche, elle a enjoint le gouvernement irlandais à prendre les mesures nécessaires pour déterminer son degré de responsabilité et réparer les dommages causés. En parallèle, Justice For Magdalens s'est adressée au Comité contre la Torture des Nations Unies afin que soit examinée la responsabilité de l'État irlandais dans les traitements inhumains subis par les anciennes pensionnaires des Magdalen Laundries Le rapport du Comité est accablant. Il n'est désormais plus possible pour l'État de nier sa responsabilité dans cette période trouble et sombre de son histoire. Le scandale des Magdalen Laundries relève désormais des droits de l'homme et, de fait, le gouvernement irlandais doit désormais envisager de rendre justice aux anciennes pensionnaires.

Mots clés : Magdalen Laundries, Église catholique, État irlandais (République d'Irlande), droits de l'homme, Commission des Droits de l'Homme irlandaise, Justice For Magdalens.

\section{Abstract}

Since the 1990s, researchers, the media and autobiographies of former inmates have extensively covered the scandal of the Magdalen Laundries. So far, the Catholic Church has had to bear the full brunt of the accusations of degrading treatment within the institutions. In 2010, Justice For Magdalens, an organisation created to demand and obtain public excuses and a restoration scheme for

1. "In conclusion, the IHRC cannot adjudicate on a breach of human rights. We cannot definitely say that the human rights of these women and girls who resided in the laundries were breached as this is beyond our powers. The Commission has, however, come to the conclusion that there are very clear questions of human rights compliance arising from this assessment. We consider that these substantiate the need to establish a statutory enquiry mechanism to fully and thoroughly investigate the matters raised by Justice for Magdalenes ", opening remarks by IHRC Commissioner Olive Braiden, Magdalen Laundries Report launch, 9 Nov 2010, [http://www.ihrc.ieldownload/pdflopening_remarks_by_ibrc_commissioner_olive_braiden_magdalen_laundries_9_nov_2010.pdf]. 
former inmates, asked the Irish Human Rights Committee to start an inquiry into the Irish State's responsibility and involvement in admission into and abuses within the Laundries. The IHRC examined JFM's request but refused to start an inquiry based on lack of formal evidence. However, it explicitly asked the Irish State to start its own inquiry and provide a restoration process. JFM went further and addressed a request to the United Nations Committee Against Torture. The UN Committee, after examining the request, asked the Irish government for an independent investigation into the Magdalene Laundries abuse and redress for the women who suffered. Magdalen Laundries have now become a human rights issue and the government, especially the Justice Department, is being compelled to act upon UN recommendations and provide answers, excuses and restoration for former inmates.

Keywords : Magdalen Laundries, Irish State, Irish Human Rights Commission, Justice for Magdalens, human rights.

En juin 2010, une organisation irlandaise du nom de Justice for Magdalens (JFM) a pris contact avec la Commission irlandaise des Droits de l'Homme (Irish Human Rights Commission ou IHRC) pour demander qu'une enquête soit diligentée en application de l'article 9.1.b du Human Rights Commission Act voté en 2000. Cette requête devait permettre d'examiner la manière dont les jeunes filles et les femmes qui avaient séjourné dans les établissements appelés Magdalen Laundries avaient été traitées et aboutir à établir la responsabilité de l'État irlandais afin de réclamer et obtenir des excuses publiques et des compensations financières. Compte tenu du caractère sérieux et grave des allégations portées par Justice for Magdalens et du fait que bon nombre d'anciennes pensionnaires de ces institutions étaient désormais très âgées, la Commission des droits de l'homme a décidé de donner priorité à l'examen de la requête.

Ladite commission a étudié de près les documents fournis par Justice for Magdalens et a rendu un premier bilan interne en septembre 2010. Sur la base de ce bilan ${ }^{2}$, la Commission a décidé de ne pas mener l'enquête demandée par Justice for Magdalens mais d'en appeler directement à l'État irlandais afin que ce dernier examine les questions relatives aux droits de l'homme dans le cas des anciennes résidentes des institutions. La commission a ainsi décidé de rendre son bilan public et d'enjoindre immédiatement l'État à mettre en place un mécanisme statutaire afin de prendre en compte les questions soulevées par Justice for Magdalens.

Le bilan a été rendu public en novembre 2010 et, pour la première fois, la question de la responsabilité de l'État dans les mauvais traitements subis par les pensionnaires de ces Magdalen Asylums y est soulevée. Le présent article se propose de décrire et d'analyser cette évolution, d'autant plus significative que, jusque-là, seule l'Église catholique irlandaise avait été jugée responsable ${ }^{3}$.

2. Voir note 1 .

3. La Commission dite Ryan, chargée d'enquêter sur les cas d'abus et de mauvais traitements sur les Enfants 
En effet, depuis les années 1990, la réputation de l'Église catholique irlandaise est entachée de scandales divers et variés ${ }^{4}$, impliquant non seulement des prêtres mais également des religieux et religieuses travaillant au sein des institutions. Dans un premier temps, et ce jusqu'à la publication des rapports Ryan et Murphy ${ }^{5}$, c'est l'Église catholique qui a dû porter l'entière et seule responsabilité des dysfonctionnements et mauvais traitements subis au sein des institutions en général, exonérant ainsi le monde politique et l'État. Or, comme l'ont démontré les Commissions Ryan et Murphy, l'État, par le biais du Ministère de l'Éducation, a autorisé que se mette en place un régime quasi carcéral au sein des institutions de prise en charge des groupes dits vulnérables puisqu'aucune mesure de supervision ou inspection efficace n’a été prise. Les congrégations religieuses et les écoles ont été protégées et couvertes pendant des décennies. La Commission a été contrainte d'admettre que les enfants avaient été victimes de traitements inhumains et cruels au sein des institutions et que cela ne relevait plus exclusivement de la responsabilité de l'Église Catholique.

C'est précisément cette question fondamentale qui est soulevée par la requête de Justice for Magdalens et qui a été examinée par l'IHRC.

L'angle d'approche est également fort intéressant dans la mesure où, pour la première fois, en déplaçant la responsabilité des institutions religieuses vers la sphère publique et politique, il est enfin possible d'envisager que la question du respect ou non-respect des droits de l'homme puisse être abordée sur le plan légal et par une instance internationale.

Dans un premier temps, nous reviendrons brièvement sur les origines et la nature des institutions dont il est question, à savoir les Magdalen Laundries ou Magdalen Asylums. Nous montrerons comment ces institutions se sont inscrites dans une perspective historique liée à la progression de l'Irlande vers son indépendance. Nous montrerons également comment l'Église catholique s'est progressivement substituée à l'État dans la prise en charge des groupes de la société perçus comme vulnérables, permettant le développement d'un vaste réseau d'institutions gérés par les congrégations religieuses.

La seconde partie de cet article sera consacrée à l'analyse de la requête de Justice for the Magdalens et des relevés de conclusions de la Commission des Droits de l'Homme.

(The Commission to Inquire Into Child Abuse), a été mise en place en 1999 et a rendu public son rapport en mai 2009. Dans ce rapport, elle a clairement dénoncé la responsabilité de l'État Irlandais dans les mauvais traitements subis par les enfants au sein d'institutions. Un processus d'indemnisation des victimes est en cours mais les modalités restent encore à définir et la question de la contribution des congrégations religieuses n’est pas réglée.

4. Cet aspect sera développé dans la première partie de cet article.

5. Voir note 3 supra. 
En conclusion, nous proposerons un bilan qui rendra compte des forces et les faiblesses des conclusions de l'IHRC et examinerons les perspectives futures.

\section{Les Magdalen Laundries : perspective historique et évolution}

Au début des années 1990, un certain nombre de scandales portèrent un coup sévère à l'institution de l'Église catholique en Irlande. De révélations sur des cas de prêtres pédophiles ${ }^{6}$, aux abus subis par des enfants pendant des décennies au sein d'institutions, ces événements marquèrent sans aucun doute le début de la " diminution spectaculaire de la pratique religieuse, le déclin marqué des vocations et la perte quasi totale d'autorité de l'institution de l'Église ${ }^{7}$ ». À la suite de ces affaires, les médias s'emparèrent de la question et plusieurs documentaires furent réalisés sur les institutions de prise en charge des femmes et des enfants ${ }^{8}$. En 1993, lorsque Les Sœurs de Notre-Dame de la Charité du Refuge, contraintes pour des raisons économiques, de vendre à un promoteur immobilier le terrain sur lequel avait été construite l'une des plus grandes institutions du pays, le Magdalen Asylum de High Park, à Drumcondra ${ }^{9}$, demandèrent au Ministère de l'Environnement un certificat d'exhumation car 177 pensionnaires y étaient enterrées, on découvrit les corps de 80 pensionnaires dont le décès n'avait pas été signalé et 22 corps de pensionnaires anonymes, mortes entre 1960 et 1970.

En 2002, la sortie du film de l'acteur et réalisateur écossais, Peter Mullan, The Magdalen Sisters, conféra une dimension internationale à cette page douloureuse de l'histoire irlandaise. Le film, très chaleureusement accueilli par le public irlandais, européen et américain, provoqua un tollé au sein de l'Église catholique, en Irlande et dans le monde. À la suite de ce film, de nombreux ouvrages autobio-

6. On peut citer le cas de l'évêque Eamon Casey, accusé d'avoir eu un fils avec une femme qui travaillait pour lui et d'avoir utilisé les deniers de l'Église pour acheter le silence de la mère, celui de Brendan Smyth, arrêté et jugé pour des affaires de pédophilie, entre autres.

7. "The dramatic fall-off of religious practice, the steep decline in vocations to the religious life and the near total loss of authority by the institutional church" Conor Brady, Irish Times, 8 octobre 2005.

8. Louis Lentin, Dear Daughter, RTE 1996, funded by RTÉ and Bord Scannan na hEireann (The Irish Film Board). 1997 Banff Television Festival Nomination for Social and Political Documentary, 1996 Creative Excellence Certificate, US International Film and Television Festival. Ce documentaire relatait l'expérience de Christine Buckley au sein de l'orphelinat de Goldenbridge, géré par les Sœurs de la Miséricorde, dans les années 50 et 60. Mary Raftery, States of Fear, RTE, 1999, série en trois parties, qui révéla les abus physiques et psychologiques auxquels furent soumis des générations d'enfants au sein du système des Industrial Schools, sortes de maisons de redressement, entre 1930 et 1970 . Raftery publia, la même année, un ouvrage, Suffer The Little Children, qui déclencha une série d'enquêtes et aboutit à la mise en place de La Commission to Inquire into Child Abuse en 2000 dont le rapport, aussi connu sous le nom de Ryan Report, fut publié en mai 2009.

9. Le Magdalen Asylum de High Park, Drumcondra à Dublin ouvrit ses portes en 1833 selon G. D. Williams (Dublin Charities, Being a Handbook of Dublin Philanthropic Organisations and Charities, Compiled and Published by the Association of Charities, Dublin : 1902) et 1853 selon Rosa M. Barrett (A Guide to Dublin Charities, 1884). 
graphiques relatant les expériences de femmes ayant séjourné dans des institutions similaires furent publiés ${ }^{10}$.

Si les prémices du " madeleinisme » en Irlande étaient de nature philanthropique, petit à petit, faute de financement, et l'enthousiasme des bienfaiteurs ayant diminué, la plupart des institutions ont été confiées à des ordres religieux, leur conférant une nouvelle dimension. La première institution irlandaise ouvrit ses portes à Dublin en 1767 sous l'égide d'une philanthrope protestante du nom d'Arabella Denny. Elle avait travaillé à l'Hôpital des Enfants Trouvés de Dublin et, inspirée par le modèle du Magdalen Hospital créé à Londres en $1758^{11}$, avait à son tour décidé d'ouvrir un refuge pour accueillir les femmes qui avaient dû abandonner leurs enfants, souvent illégitimes. De nombreux refuges protestants et catholiques furent établis à la même époque mais tous étaient gérés par des laïcs. Ils s'appelaient Magdalen Refuges ou Magdalen Hospitals ${ }^{12}$.

Au sein de ces institutions, la femme était envisagée en tant que corps-objet sur lequel s'exerçait une forte projection symbolique. Son corps qui n'était plus intact était un objet de scandale en ce qu'il représentait le lieu et la mémoire d'une transgression, même lorsque la femme n'était pas considérée comme responsable de son déshonneur. Par conséquent, le devoir premier des institutions qui prenaient en charge les femmes déchues mais également les femmes dites " en danger " était de faire disparaître le corps-objet qui constituait la mémoire du scandale. En ce sens, les institutions et l'idéologie qui prévalaient dans l'Europe catholique influencèrent indéniablement le développement du système " madeleiniste ", tel qu'il se développa en Irlande. Il ne faut cependant pas perdre de vue que les philanthropes irlandais s'étaient inspirés de leurs homologues anglais qui, eux-mêmes s'étaient inspirés d'institutions qui existaient dans l'Europe catholique dès le treizième siècle.

Dès 1830 , un vaste réseau conventuel se développa en Irlande, lié à l'implantation de congrégations religieuses féminines ${ }^{13}$. Lorsque les institutions de prise en

10. Goulding, June, The Light in the Window, Dublin, Poolbeg, 1998. O'Malley, Kathleen, Childhood Interrupted, Virago, 2005. O’Beirne, Kathy, Kathy's Story, Edinburg, Mainstream Publishing, 2005. La liste n'est pas exhaustive.

11. Inspiré par les institutions catholiques des $\mathrm{XVI}^{\mathrm{e}}$ et $\mathrm{XVII}^{\mathrm{e}}$ siècles des philanthropes anglais. On peut notamment citer Jonas Hanway et Robert Dingley, deux marchands qui avaient parcouru le monde et observé le fonctionnement des entreprises caritatives dans l'Europe catholique. Membres d'un groupe de philanthropes, ils fondèrent le Magdalen Hospital de Londres. Au sein de cet établissement, les prostituées étaient prises en charge corps et âme. On leur enseignait un métier, on les soignait, physiquement et moralement.

12. Deux ouvrages recensent les institutions existant à Dublin à la fin du dix-neuvième siècle:Williams, G. D., Dublin Charities, Being a Handbook of Dublin Philanthropic Organisations and Charities, Compiled and Published by the Association of Charities, Dublin, 1902 et Barrett, Rosa M., A Guide to Dublin Charities, 1884, NLI.

13. The Sisters of Mercy, en anglais. Ordre fondé en 1832 par Catherine McAuley. The Sisters of Charity, en anglais. Ordre fondé par Mary Aikenhead. The Good Shepeherd Sisters ou Soeurs de Notre-Dame de Charité du BonPasteur, ou Sœurs du Bon-Pasteur, en français. Cette congrégation fut fondée à Angers en 1835 par sainte Marie-Euphrasie Pelletier. 
charge des femmes vulnérables furent confiées à ces congrégations, la perception et représentation des pensionnaires changea. Soucieux de lutter contre le prosélytisme des anglicans, les catholiques commencèrent à envisager la vulnérabilité comme un comportement moral déviant et à instaurer un régime plus répressif et punitif. Ce mouvement ne fit que croître et se renforcer pour atteindre son paroxysme avec l'avènement de l'État Libre.

À la suite des mouvements pour l'indépendance de l'Irlande et au moment de la création de l'État Libre, le rôle et le statut de l'Église catholique dans le domaine de la prise en charge des groupes perçus comme vulnérables dans la société devint de plus en plus important. Ainsi, les Magdalen Asylums accueillirent de moins en moins de prostituées et de plus en plus de mères célibataires ou de jeunes filles perçues comme en danger moral. En outre, la volonté du jeune État de se démarquer de l'ex-occupant britannique renforçait cet attachement aux enseignements de l'Église et les discours politiques des trois premières décennies du vingtième siècle étaient fondamentalement indissociables des enseignements et valeurs du catholicisme. Les femmes furent au cour de la construction identitaire de l'État Libre ${ }^{14}$, une identité nouvelle, religieuse et morale qui devait s'affranchir de l'abâtardissement dont elle avait été victimes ${ }^{15}$. Dès les années 1920 , une série de lois discriminatoires à l'égard des femmes furent votées, influencées par les enseignements sociaux de l'Église, qui renvoyèrent les femmes à leur statut de mères et d'épouses ${ }^{16}$. Ces lois devaient $s^{\prime}$ inscrire dans la volonté de l'Irlande de se désolidariser de la Grande-Bretagne et de mettre en exergue ses valeurs morales.

C'est précisément dans ce contexte que les Magdalen Asylums accueillirent de plus en plus de pensionnaires. Ils permettaient de cacher des femmes qui, par leur comportement perçu comme immoral, troublaient l'ordre social. L'État se déchargeait ainsi à la fois de la responsabilité financière ${ }^{17}$, morale et sociale des individus dont il jugeait qu'ils devaient être cachés qu'il jugeait qu'il convenait de cacher. C'est ce que James Smith a appelé "l'architecture irlandaise d'endiguement ». Voici comment il définit ce concept :

Dans sa forme concrète, cette architecture englobait un réseau d'institutions interdépendantes parmi lesquelles les maisons maternelles, les

14. Pour une analyse de cet argument, voir Maryann Valiuilis, « Neither Feminst Nor Flapper : The Ecclesiastical Construction of the Ideal Iirish Woman, in Mary O'Dowd and Sabine Wicher (eds), Chattel, Servant or Citizen? Women's Status in Church, State and Society, Belfast, 1995, p. 168-178.

15. Pour une analyse de la construction identitaire de l'État Libre, voir Richard Kearney, On Stories, London, Routledge, 2002.

16. Lois sur les Jurés (1924 et 1927) ; Loi sur la Réglementation de la Fonction Publique (1925); Loi sur la Censure des Publications (1929); Loi sur les Enfants Illégitimes et la Constatation de Paternité (1930).

17. Le financement des institutions était en effet assuré par le travail des pensionnaires qui y avaient le linge des hôtels, écoles, universités et autres institutions, contrairement à d'autres institutions comme les Industrial Schools qui bénéficiaient de subventions per capita. 
agences d'adoption, les Magdalen Asylums, les Industrial Schools et autres maisons de redressement. Oeuvrant conjointement, ces sites d'incarcération cachaient au regard public les éléments les plus indésirables relevant des trois phénomènes sociaux suivants : l'adoption, l'infanticide et la prise en charge des enfants en établissements spécialisés. Dans sa forme plus abstraite, cette architecture comprenait aussi, à la fois la législation qui définissait ces trois problèmes, et de nombreux discours, officiels et publics, qui refusaient d'admettre l'existence et la fonction des institutions chargées de régler ces questions ${ }^{18}$.

Il est très difficile de savoir précisément combien de femmes ont séjourné dans ces refuges puisqu'aucun registre n'est disponible ou accessible. Les congrégations religieuses refusent encore, à ce jour, de les rendre publics. En outre, comme l'a révélé la Commission Ryan ${ }^{19}$ dans son rapport accablant, les Magdalen Asylums ont toujours réussi à échapper à toute forme de contrôle et d'inspection. L'une des raisons qui expliquent cette situation inédite est qu'officiellement, les femmes y entraient volontairement ${ }^{20}$. Il ne s'agissait donc pas de lieux d'incarcération mais de refuges pour des âmes en perdition.

Depuis les années 1990, néanmoins, d'anciennes pensionnaires et leurs enfants se sont organisés afin que leur histoire soit désormais inscrite dans l'histoire nationale et que soit enfin levé le sceau du secret qui entoure cette douloureuse période.

\section{Analyse de la requête de Justice for Magdalens et du rapport de l'Irish Human Rights Commission}

Avant de présenter les conclusions de la Commission, il convient de revenir sur la requête déposée par Justice for Magdalens et sur la nature même de cette organisation.

18. «In its concrete form, this architecture encompassed an array of interdependent institutions - schools, hospitals, mother-and-baby homes, adoption agencies, and Magdalen laundries - that obscured the less desirable elements attached to a number of interrelated social phenomena, including poverty, illegitimacy, and infanticide. In its more abstract form, this architecture comprised both the legislation that inscribed these issues as well as the numerous official and public discourses that resisted admitting the existence and function of their affiliated institutions ", James M. Smith, Ireland's Magdalen Laundries and the Nation's Architecture of Containment, Notre Dame, Indiana, University of Notre Dame Press, 2007, p. 2.

19. The Commission to Inquire Into Child Abuse (voir note 3). Le Volume 3, Chapitre 18 est intitulé "Residential Laundries, Novitiates, Hostels and other Out-of-home Settings ". À aucun moment, le terme Magdalen Laundries n'est mentionné. Même si la Commission était sensée s'intéresser aux conditions d'incarcération des enfants et adolescents, il est très surprenant que ces institutions n'y aient pas une plus grande place. Le terme Magdalen n'est jamais utilisé. Par conséquent, ces institutions n’ont pas été prises en compte dans le projet de réparation et compensation de 2002, Residential Institutions Redress Act, 2002.

20. Rebecca Lea McCarthy, Origins of the Magdalen laundries : an analytical history, McFarland, 2010, p. 197-198. 


\section{La requête de Justice for Magdalens}

\section{Origines de l'organisation}

Justice for Magdalens se présente ainsi : "Justice for Magdalenes is comprised of survivors, the family members of survivors, long-time activists in human rights and adoption reform, academics, researchers, archivists and representatives from the political community ${ }^{21}$."

Le groupe a été créé en 2004 et est l'émanation d'une organisation antérieure, le Magdalen Memorial Committee, formé en 1993, qui s'était engagé dans une campagne afin d'obtenir qu'un monument honore les 133 femmes enterrées sur le terrain du Magdalen Asylum de High Park, à Drumcondra, puis exhumées, incinérées et inhumées au cimetière de Glasnevin ${ }^{22}$. Les révélations de ces procédures, sans doute peu légales mais qui n'ont fait l'objet d'aucune enquête approfondie, ni par la police ni par les politiques, ont ému l'opinion publique, et des femmes, certaines étant les enfants adoptées d'anciennes résidentes des institutions, ont continué leur engagement et fondé Justice for Magdalens.

Le groupe a pour objectifs principaux d'obtenir des excuses officielles de la part de l'État irlandais et de l'Église catholique et d'obtenir, également, que soit mis en place un système de réparation spécifique aux survivantes des Magdalen Laundries. À ce jour, aucune excuse publique ni aucun mécanisme juridique n'existe pour défendre les intérêts des anciennes pensionnaires.

\section{La campagne de Justice for Magdalens}

En septembre 2009, Justice for Magdalens s'est adressée au ministre de l'Éducation de l'époque en lui demandant des excuses et un projet de réparation pour les anciennes pensionnaires de Magdalen Laundries. Le groupe s'est vu opposer une fin de non-recevoir pour les mêmes raisons que celles invoquées pour le Redress $A c t^{23}$.

Justice for Magdalens a ensuite rencontré le Cardinal Sean Brady en 2010, afin d'impliquer les autorités religieuses dans leur campagne. Ce dernier les a encouragés à continuer leurs efforts pour établir un dialogue et un processus de justice et réconciliation pour toutes les parties concernées et leur a même recommandé de rencontrer la Directrice Générale de CORI, The Conference of Religious of Ireland ${ }^{24}$. La directrice a refusé de rencontrer Justice for Magdalens tout en

21. [www.magdalenelaundries.com/about.htm]. Dernière consultation le 21 janvier 2012.

22. “Taking Mary Home”, Mary Raftery, Irish Times, 15 avril 2004.

23. Irish Times, 2 mai 2011.

24. CORI (Conference of Religious Of Ireland) ou La Conférence des Religieux d'Irlande a pour but de servir les dirigeants et à travers eux les membres des congrégations religieuses. Il fournit un forum où les religieux peuvent travailler ensemble dans la mission qu'ils ont en commun, [http://www.cori.ie/religiouslife/missionstatement], dernière consultation le 30 avril 2012. 
conseillant à l'organisation de prendre directement contact avec les congrégations religieuses impliquées. Toutes les tentatives ont été infructueuses et inabouties ${ }^{25}$.

Poursuivant sans relâche ses efforts, le groupe a adressé sa requête à la Commission des Droits de l'Homme en juin 2010²6, afin que celle-ci examine un certain nombre de questions qui pourraient représenter des cas de non-respect des droits de l'homme. La requête soulevait de nombreux points mais nous allons, ici, nous attacher à trois d'entre eux qui nous ont semblé fondamentaux.

\section{Les questions soulevées par Justice for Magdalens}

Le premier point soulevait la question du degré d'implication et de responsabilité de l'État irlandais dans les circonstances qui avaient permis que des jeunes filles et des femmes soient admises dans ces institutions.

Dans le deuxième point, Justice for Magdalens demandait à la Commission de se prononcer sur la notion de travail forcé et de servitude, compte tenu que les pensionnaires fournissaient un travail pour lequel elles n'étaient pas rétribuées.

Le troisième et dernier point invitait la Commission à se prononcer sur la possibilité pour les enfants illégitimes abandonnés sous la contrainte par les résidentes des institutions, puis adoptés, de retrouver la trace de leur mère comme le prévoit la législation européenne en vigueur.

Ces questions, fondamentales, permettent de situer le débat sur une nouvelle échelle. En effet, jusque-là, seule la question de la responsabilité de l'Église et des congrégations religieuses avait été soulevée. Or, en invoquant la question d'un potentiel non-respect des droits de l'homme, Justice for Magdalens fait glisser le débat vers une responsabilité de l'État et entre dans une bataille juridique.

\section{L'examen de l'IHRC et ses conclusions}

Le rapport de la Commission comprend douze relevés de conclusions et pas moins de 108 paragraphes. Il n'est bien entendu pas question de tous les examiner mais de s'arrêter sur ceux qui répondent aux questions fondamentales que nous venons de souligner.

1- Concernant le degré d'implication et la responsabilité de l'État dans le processus d'admission des pensionnaires au sein des institutions, la commission relève que Justice for Magdalens a fourni des preuves incontestables (sous la forme d'archives des tribunaux) que dans trois cas, au moins, l'État irlandais, par le biais de son système judiciaire, plus précisément ses tribunaux, avait autorisé que des femmes condamnées pour des meurtres, des homicides involontaires ou des infanticides voient

25. [http://www.magdalenelaundries.com/newsletters/newsletter_v1_7.pdf]. Dernière consultation le 21 janvier 2012. 26. [http://www.magdalenelaundries.com/press/JFM\%20PR\%2024-06-10.pdf]. Dernière consultation le 21 janvier 2012. 
leur peine commuées à des peines avec sursis à condition qu'elles résident dans des Magdalen Laundries. Il pouvait s'agir de femmes condamnées à une peine précise, de femmes placées en liberté surveillée ou en détention préventive. Dès 1936, le Rapport Cussen ${ }^{27}$ avait demandé que cette pratique soit inscrite dans la législation et que les institutions soient rétribuées pour le service rendu. Cela prouve bien que l'État « trouvait son compte » dans cette pratique et y participait.

En outre, des preuves indiquent que l'État subventionnait quelques blanchisseries qui accueillaient des femmes en liberté surveillée ou en préventive. Le Rapport Kennedy ${ }^{28}$, publié en 1970, qui fut l'un des premiers sur les conditions de vie au sein des maisons de redressement et des Industrial Schools offre un éclairage intéressant :

A number of [girls] considered by parents, relatives, social workers, Welfare Officers, Clergy or Gardai to be in moral danger or uncontrollable are also accepted in these convents for a period on a voluntary basis. From enquiries made, the Committee is satisfied that there are at least 70 girls between the ages of 13 and 19 years confined in this way who should properly be dealt with under the Reformatory School's system ${ }^{29}$.

Cet extrait illustre bien le fait que même si de nombreux agents privés étaient responsables de l'admission des filles au sein des Magdalen Laundries, l'État était également impliqué dans la mesure où la police et les travailleurs sociaux pouvaient les faire admettre aussi.

De surcroît, la terminologie utilisée pour qualifier les jeunes filles indique le souci des autorités de souligner le caractère moral du choix. Il s'agissait de filles dites incontrôlables ou en danger moral, ce qui renvoie à la double fonction de ces institutions, à savoir préventive et punitive. Dans un souci de protéger ces jeunes filles d'elles-mêmes, on les enfermait afin de s'assurer qu'elles ne contamineraient pas la société.

Ainsi, la Commission des Droits de l'Homme constate, dans sa première conclusion que l'État ne peut nier avoir collaboré et participé à l'admission d'une certaine catégorie de jeunes filles et de femmes dans les Magdalen Laundries.

2- La seconde question concerne la manière dont les pensionnaires, ou résidentes, ou encore pénitentes, étaient traitées au sein des institutions. Pour Justice

27. Commission of Inquiry into the Reformatory and Industrial School System 1934-1936 chaired by Justice Cussen. C'est ce rapport qui a servi de point de départ à la Commission Ryan.

28. Reformatory and Industrial Schools System Report, 1970, Dublin : Stationery Office. La Commission, présidée par le Juge Eileen Kennedy, s'intéressa de près à la prise en charge des enfants notamment au sein des institutions et parvint à la conclusion que le système existant était insatisfaisant et avait grand besoin d'être amendé et amélioré.

29. Ibid., p. 38, 6.18. 
for Magdalens, elles ont subi des mauvais traitements, ce qui justifie une enquête plus approfondie. Dans le chapitre 18 du Rapport Ryan ${ }^{30}$, la Commission cite des témoignages relatifs aux mauvais traitements subis par de très jeunes filles au sein de "residential laundries ». Pour autant, la définition de ces institutions étant peu claire et précise, la Commission des Droits de l'Homme considère qu'elle ne peut se prononcer sur le fait qu'il s'agissait bien de Magdalen Laundries et non de blanchisseries d'État, même si, à la lecture des témoignages, il est clairement et souvent fait mention de sœurs et de religieuses.

La commission note, par ailleurs, que les preuves relatives à ces circonstances dépendent exclusivement des témoignages d'anciennes résidentes, relatés dans le rapport, et dans un documentaire de la BBC. Ce qui nous renvoie à la question des sources et des traces sachant que toutes les archives relatives aux Magdalen Laundries sont entre les mains des congrégations religieuses, ou du moins c'est ce que l'on suppose, et que toute tentative d'y avoir accès est vaine.

3- Le troisième point concerne la question du travail au sein des Magdalen Laundries. La Commission note que les résidentes travaillaient pendant des heures dans des conditions difficiles dans les blanchisseries des institutions et que les couvents qui géraient les blanchisseries bénéficiaient financièrement de cet arrangement. En signant la Convention sur le Travail Forcé en 1930, le gouvernement irlandais s'est engagé à prohiber toute forme de travail forcé ou obligatoire. Cette convention comporte des exceptions, notamment concernant les individus sous le coup d'une condamnation, mais le travail fourni doit l'être sous la supervision des autorités compétentes. Or, si des pensionnaires de Magdalen Laundries condamnées à des peines de prison avec sursis pouvaient, en vertu de cette législation, être contraintes de travailler dans les blanchisserie sans être rémunérées, l'obligation de supervision et de contrôle exigé par la loi n’a pas été respectée par les autorités puisque jamais aucun contrôle n'a été effectué au sein de ces établissements par des agents de l'État. Pour autant, en l'absence de preuves ou de documents, la Commission ne peut qu'émettre des suppositions et avancer que l'État irlandais pourrait ne pas avoir respecté la convention de 1930.

4- La question de l'adoption est au centre des préoccupations de Justice for Magdalens dans la mesure où, comme nous l'avons souligné, l'organisation est en partie composée d'enfants adoptés dont les mères biologiques sont d'anciennes pensionnaires des Magdalen Laundries. La première disposition législative relative à l'adoption en Irlande date de 1952. Mais bien avant que cette loi ne fût votée, un grand nombre d'enfants, très souvent des enfants dits illégitimes, avaient

30. Voir note 18 supra. 
été adoptés de manière tout à fait informelle, notamment par des familles américaines et australiennes. Le certificat de naissance portait le nom des parents adoptifs, effaçant toute trace des origines biologiques. Avant le vote de la première loi sociale pour les mères célibataires en 1973, l'État préférait subventionner le placement des mères et des enfants illégitimes dans des Mother and Baby Homes ou le placement des enfants dans des institutions. Ces Mother and Baby Homes étaient, également, des agences d'adoption, et la Commission se demande, à juste titre, si le consentement de la mère, tel qu'il était requis par la loi de 1952, était demandé et obtenu. Les possibilités d'une mère célibataire de s'occuper et d'élever son enfant à cette époque - sans compter les implications morales - étaient si limitées que la mère n'avait souvent guère le choix que de consentir à l'adoption. Il faut également savoir que l'État finançait les Mother and Baby Homes avec un système d'allocation per capita ${ }^{31}$. Lorsque les enfants avaient été adoptés, les mères étaient souvent transférées dans des Magdalen Laundries. Dans ces conditions, il est très difficile aujourd'hui pour les enfants adoptés de retrouver la trace de leur mère biologique. Or, cela contrevient aux dispositions de la Convention Européenne des Droits de l'Homme, ratifiée par l'Irlande, en vertu de laquelle, si la mère biologique est présumée décédée, son identité peut être révélée à l'enfant (alors que si elle est en vie son droit à la vie privée est supérieur au droit de l'enfant adopté). La Commission considère dès lors que compte tenu que de nombreuses femmes sont mortes au sein de Magdalen Laundries, les enfants devraient pouvoir connaître leur identité. Pour autant, les congrégations religieuses n'ont pas jugé bon de produire ces documents et les traces de ces femmes ont été, en quelque sorte, effacées. Aucune trace d'acte de naissance ou de certificat de décès n'est disponible. Dans le paragraphe 93 de son rapport, la Commission souligne que ces documents devraient pouvoir être accessibles et que même si tel n’a pas été le cas jusque là, les adoptés pourraient en référer à la Cour Européenne des Droits de l'Homme.

D'autres questions sont soulevées par Justice for Magdalens, et examinées par la Commission dans son rapport, mais il serait trop long de toutes les présenter ici et elles découlent des questions fondamentales que nous avons choisi d'aborder.

Quoi qu'il en soit, après examen, la Commission a conclu que des questions restent sans réponse et que des infractions ont pu être commises par l'État irlandais. Elle recommande, par conséquent, que ce dernier se penche sur ces questions et prévoit un mécanisme statutaire à appliquer s’il apparaissait que des dysfonctionnements existent.

Elle enjoint donc l'État irlandais à se pencher sur la question de sa responsabilité dans les procédures d'admission des pensionnaires des Magdalen Laundries,

31. James M. Smith, Ireland's Magdalen Laundries and the Nation's Architecture of Containment, Notre-Dame, Indiana, University of Notre Dame Press, 2007, p. 52. 
et d'enquêter sur les conditions de vie de ces femmes au sein des institutions. Si la responsabilité de l'État était avérée, ce dernier devrait alors prendre en compte ces éléments et, en collaboration avec les anciennes résidentes et les groupes de soutien, mettre en place un processus de réparation.

\section{Quel bilan pouvons-nous faire de ces conclusions?}

Justice for Magdalens s'est déclarée satisfaite des recommandations faites par l'IHRC et a poursuivi sa campagne de manière très assidue et suivie. Dr. Katherine O'Donnell, la Directrice du Women's Studies Centre de UCD, et membre du Comité consultatif de Justice for Magdalens, a déclaré :

In the midst of an economic crisis that seems to challenge the sovereignty of our state, Irish citizens are daily asking - what kind of social values do we want, what kind of society do we want our children to inherit? In the spirit of these concerns, JFM asks this current government to show the leadership requested by the IHRC; and to immediately apologise and begin the process of acknowledging and ultimately understanding our very recent dark history ${ }^{32}$.

Le gouvernement, par le biais de son ministre de la Justice, a déclaré être disposé à prendre ces recommandations en compte, et a reconnu que le fait que les Magdalen Laundries étaient des institutions privées ne dispensait pas l'État de régler, une fois pour toutes, la question II a précisé qu'une décision serait prise avant Pâques $2011^{33}$.

Le 3 mai 2011, Justice for Magdalens a déposé une requête devant le Comité des Nations Unies contre la Torture afin que pour la première fois, l'Irlande ait à répondre de traitements inhumains sur les pensionnaires de Magdalen Laundries ${ }^{34}$.

Le 3 juin 2011, le Comité contre la torture des Nations Unies (UNCAT) ${ }^{35}$ a remis ses conclusions ${ }^{36}$. Elles sont assez accablantes. Le Comité regrette en premier lieu les réductions budgétaires liées au financement de l'IHRC ainsi que sa récente affiliation au ministère de la Justice. Le Comité des Nations Unies recommande ainsi à l'État irlandais de s'assurer que « les coupes budgétaires affec-

32. [http://www.magdalenelaundries.com/press/JFM\%20PR\%2009-11-10.pdf]. Dernière consultation le 21 janvier 2012.

33. Ibid.

34. [http://www.magdalenelaundries.com/jfm_comm_on_torture_210411.pdf]. Dernière consultation le 21 janvier 2012.

35. Le Comité contre la torture est un organe composé d'experts indépendants qui surveillent l'application de la Convention contre la torture et autres peines ou traitements cruels, inhumains ou dégradants par les États parties.

36. [http://www.magdalenelaundries.com/press/JFM\%20PR\%2005-06-11.pdf]. Dernière consultation le 21 janvier 2012. 
tant actuellement les organismes des Droits de l'Homme, et particulièrement l'IHRC, ne paralysent pas leurs activités ni ne rendent leur mandat inefficace ${ }^{37}$ ".

Concernant spécifiquement les Magdalen Laundries, le Comité est clair et se déclare très préoccupé que l'État n'ait pas protégé les jeunes filles et femmes enfermées contre leur gré dans des Magdalen Laundries entre 1922 et 1996. Le Comité affirme également que l'État n'a pas garanti la mise en place de structures d'inspection, alors qu'il est avéré que les pensionnaires y ont subi de mauvais traitements physiques et émotionnels qui contreviennent à la Convention des Droits de l'Homme. Par conséquent, le Comité recommande que l'État partie diligente des enquêtes rapides, indépendantes et complètes sur les accusations de torture et autres traitements ou punition cruels, inhumains ou dégradants qui auraient été commis dans les Magdalen Laundries et, le cas échéant, qu'il poursuive et en punisse les auteurs. Il recommande également que les victimes obtiennent réparation et compensations financières pour les préjudices subis ${ }^{38}$. Dans le paragraphe 33 du relevé de conclusions, le Comité des Nations Unies précise que l'État irlandais dispose d'un délai d'un an pour mettre à la disposition du Comité des preuves qu'il a pris des mesures pour mettre en ouvre les recommandations formulées dans les paragraphes 8 et 21 .

\section{Comment le gouvernement a-t-il réagi à ces recommandations?}

Le 14 juin, il a publié une déclaration dans laquelle il s'engage à mettre en place un comité interdépartemental qui sera chargé de faire le lien entre l'État et les autorités religieuses, notamment CORI, afin de clarifier " la nature des interactions entre l'État et les Magdalen Laundries ${ }^{39}$ ". En parallèle, le ministère de la Justice s'engage à rencontrer les congrégations religieuses pour leur demander, et ceci est tout à fait inédit et très important, de "mettre à la disposition des anciennes pensionnaires des Magdalen Laundries et des chercheurs tous les registres et archives dont elles disposent ${ }^{40} »$. En outre, le gouvernement demande que soit engagée une réflexion avec les congrégations religieuses et les groupes de survivantes sur la mise en place d'un processus de réparation et réconciliation ${ }^{41}$.

37. Committee against Torture, Forty-sixth session 9 May-3 June 2011, Consideration of reports submitted by States parties under article 19 of the Convention, paragraphe 8.

38. United Nations Committee Against Torture, Forty-sixth session 9 May-3 June 2011, Consideration of reports submitted by States parties under article 19 of the Convention, paragraphe 21.

39. Government Statement on the Magdalene Laundries, paragraph 1, "to clarify any State interaction with the Magdalene Laundries", [http://www.magdalenelaundries.com/government_statement_laundries.pdf]. Dernière consultation le 21 janvier 2012.

40. Ibid., paragraph 2.a

41. Ibid., paragraph 2.C. 
Le 9 septembre 2011, Justice for Magdalens a rencontré pour la première fois le comité interdépartemental mis en place par le gouvernement. L'association qualifie la rencontre de fructueuse.

Le 14 octobre 2001, JFM a soumis au ministre de la Justice un projet visant à établir un processus de réparation et compensation pour les victimes ${ }^{42}$.

Le 26 octobre 2011, le comité interdépartemental a publié un rapport intérimaire dans lequel il précise que son rôle consiste exclusivement à établir les faits et qu'il ne saurait, en aucun cas, faire ou recommander que des excuses soient faites. De même, il ne se considère pas compétent pour statuer sur des cas particuliers ou proposer des mesures compensatoires ${ }^{43}$. Ce comité poursuit actuellement son travail dont le bilan devrait être rendu public en mai 2012.

Même s'il apparaît que la Commission des Droits de l'Homme a été un peu " frileuse " dans son rapport et qu'elle manquait d'informations précises lui permettant de se prononcer sur les violations potentielles des droits de l'homme, elle a refusé de s'engager à approfondir son enquête. Elle a, en outre, délibérément occulté une dimension essentielle, qui est celle de l'accès aux registres des institutions. Elle soulève la question deux fois sans pour autant reprendre ce point dans les recommandations. Or, il semblait évident qu'avant de lancer quelque processus de réparation que ce fût, il était indispensable d'enjoindre, voire de contraindre les congrégations religieuses à mettre à la disposition des autorités et du public ces documents qui, seuls, pouvaient témoigner de l'existence et des traumatismes subis par les anciennes pensionnaires. L'efficacité et l'indépendance de la Commission Irlandaise des Droits de l'Homme ont fait l'objet d'un certain nombre de controverses, notamment liées à sa composition et à ses liens étroits avec le ministère de la Justice. En outre, la Commission ne dispose pas de fonds illimités, ce qui retarde les enquêtes qu'elle engage. On peut néanmoins affirmer que ses conclusions, dans le cas des Magdalen Laundries, ont encouragé Justice for Magdalens à faire pression sur l'État et à s'adresser aux Nations Unies. Dans la mesure où la question du non-respect des droits de l'homme a été abordée et envisagée, il est devenu possible de remettre en question une lecture unilatérale de cet enjeu, renvoyant essentiellement la responsabilité à l'Église catholique et de contraindre l'État irlandais et le gouvernement à prendre leurs responsabilités.

Pour autant, s'il est désormais avéré que la perpétuation d'un tel système a été rendue possible par la collusion entre l'Église et l'État, on ne saurait négliger la question de la responsabilité de la société dans son ensemble. Les familles des jeunes filles et jeunes femmes qui ont été enfermées dans ces institutions ont, à n'en pas douter, accepté et collaboré avec un système qui leur permettait de se

42. [http://www.magdalenelaundries.com/JFM\%20Reparations\%2014\%20October.pdf]. Dernière consultation le 21 janvier 2012

43. [http://www.magdalenelaundries.com/press/JFM\%20PR\%2026-10-11.pdf]. Dernière consultation le 21 janvier 2012 
décharger de leurs responsabilités. Elles ont contribué à dissimuler l'existence d'individus qui étaient perçus comme troublant l'ordre moral qui prévalait en Irlande. Cet aspect de la question doit faire l'objet d'une étude précise afin que l'enjeu des Magdalen Asylums soit véritablement analysé et que des réponses complètes soient apportées.

Il reste désormais à poursuivre ce combat afin que les jeunes filles et femmes qui ont été victimes de ce régime répressif puissent enfin obtenir les réparations auxquelles elles ont droit. 